\title{
Long-term Nucleotide Analogue Treatment Has Higher Levels of Renal Toxicities than Does Entecavir in Patients with Chronic Hepatitis B
}

\author{
Young Youn Cho ${ }^{1,2}$, Young Chang ${ }^{1}$, Joon Yeul Nam ${ }^{1}$, Hyeki Cho ${ }^{1}$, Eun Ju Cho ${ }^{1}$, Jeong-Hoon Lee ${ }^{1}$, Su Jong Yu ${ }^{1}$, Jung-Hwan \\ Yoon $^{1}$, and Yoon Jun Kim ${ }^{1}$ \\ ${ }^{1}$ Department of Internal Medicine and Liver Research Institute, Seoul National University College of Medicine, and ${ }^{2}$ Department of Internal \\ Medicine, Chung-Ang University Hospital, Seoul, Korea
}

Background/Aims: Renal toxicity is a concern in patients with chronic hepatitis $B$ taking nucleotide analogues, such as adefovir (ADV) and tenofovir disoproxil fumarate (TDF). We sought to determine the long-term renal effects of nucleotide analogue treatment versus entecavir (ETV) treatment. Methods: In this retrospective single-center study, we selected 87 patients who were treated with ADV and subsequently with TDF from June 2008 to December 2013. ETV-treated patients were matched by treatment duration. We analyzed the creatinine increase over $0.5 \mathrm{mg} / \mathrm{dL}$, glomerular filtration rate (GFR) decrease over 25\%, phosphorus decrease under $2.0 \mathrm{mg} / \mathrm{dL}$, and dose reduction of antiviral agents. Results: The median follow-up period was 60.0 months for both groups. The incidence of liver cirrhosis was higher in the ADVTDF group than in the ETV group (32.2\% vs $74.7 \%, p<0.01)$. Creatinine increased in both groups during follow-up, but the difference was not significant $(5.7 \%$ and $2.3 \%, p=0.44)$. In addition, GFR decreased more often in the ADV-TDF group than in the ETV group (31.0\% and 14.9\%, p=0.01). After multivariate Cox regression analysis, ADV-TDF treatment was significantly associated with a GFR decrease over 25\% (hazard ratio, 2.10; 95\% confidence interval, 1.08 to $4.10 ; p=0.03$ ) after adjusting for the baseline GFR decrease. Conclusions: Patients taking nucleotide analogues had a significantly higher number of renal events than did those taking ETV. Clinicians should be aware of the development of renal toxicity in this patient population. Further long-term studies are warranted. (Gut Liver 2020;14:225-231)

Key Words: Hepatitis B, chronic; Kidney diseases; Guanine; Antiviral agents

\section{INTRODUCTION}

Chronic hepatitis B (CHB) is a major healthcare problem worldwide and a leading cause of liver-related events such as cirrhosis and hepatocellular carcinoma (HCC). ${ }^{1,2}$ Tenofovir disoproxil fumarate (TDF) and entecavir (ETV) are nucleot(s)ide analogues (NAs) with high genetic barriers that are recommended as first-line therapies in patients with $\mathrm{CHB} .{ }^{2,3}$ The key safety concern of ETV is its possible carcinogenic potential, based on preclinical rodent studies. An in vivo study showed an increased incidence of benign and malignant neoplasms involving a variety of organ sites. ${ }^{4}$ But until now, there has been no evidence of increased risk of carcinogenesis in human studies. The advantage of TDF treatment is the lower risk of HBV mutation and superior antiviral efficacy of viral suppression, compared with ETV. Major concerns of TDF treatment are renal adverse events and osteoporosis, although the nephrotoxicity has not been proved clinically significant in patients with the CHB taking TDF, compared with those taking ETV. ${ }^{5}$

TDF's mechanisms of renal toxicity in patients with human immunodeficiency virus (HIV) is thought to be mitochondrial toxicity, especially in the proximal tubule. ${ }^{6}$ Toxicity in patients with HIV and CHB manifest as Fanconi syndrome. ${ }^{6-8}$ The mechanism of ADV nephrotoxicity is also mediated by the mitochondria from proximal tubular cells and manifests as Fanconi syndrome. ${ }^{9}$ TDF and ADV also share de novo appearance of hypophosphatemia, hypouricemia, proteinuria or glycosuria, and osteomalacia. ${ }^{10}$ TDF and ADV are nucleotide analogues with similar structures, and both drugs have similar mechanisms of renal toxicity.

Previous studies on TDF renal toxicity in patients with HIV have shown that TDF renal toxicity needs a long-time interval

\footnotetext{
Correspondence to: Yoon Jun Kim

Department of Internal Medicine and Liver Research Institute, Seoul National University College of Medicine, 101 Daehak-ro, Jongno-gu, Seoul 03080, Korea

Tel: +82-2-2072-3081, Fax: +82-2-743-6701, E-mail: yoonjun@snu.ac.kr

Received on October 20, 2018. Revised on February 10, 2019. Accepted on March 1, 2019. Published online August 15, 2019. pISSN 1976-2283 eISSN 2005-1212 https://doi.org/10.5009/gnl18474

(c) This is an Open Access article distributed under the terms of the Creative Commons Attribution Non-Commercial License (http://creativecommons.org/licenses/by-nc/4.0) which permits unrestricted non-commercial use, distribution, and reproduction in any medium, provided the original work is properly cited.
} 
from the initiation of TDF treatment to appear. ${ }^{11}$ In Korea, TDF has been approved for CHB later than in other Western countries, suggesting that there may not be enough time for nephrotoxicity to appear in patients with $\mathrm{CHB}$. Since TDF and ADV share mechanisms and clinical manifestations of nephrotoxicity, we sought to evaluate renal toxicity associated with ADV and TDF versus ETV monotherapy in patients with CHB.

\section{MATERIALS AND METHODS}

\section{Patient selection}

A total of 525 CHB patients treated with TDF between Jan 2008 and December 2013 at a single university hospital (Seoul National University Hospital, Korea) were screened for this study. The inclusion criteria were: (1) age >18 years; (2) CHB patient treated with ADV, subsequent TDF; and (3) baseline glomerular filtration rate (GFR) $>30 \mathrm{~mL} / \mathrm{min} / 1.73 \mathrm{~m}^{2}$ and not receiving hemodialysis. The exclusion criteria were: (1) combined ETV-TDF treatment; (2) liver transplant prior and during antiviral treatment; (3) chemotherapy during antiviral treatment; and (4) patients with HIV or hepatitis C virus co-infection. Finally, 87 patients were included in this study, and we designated this group as the ADV-TDF group. A total of 670 patients treated with ETV monotherapy between 2008 and 2013 with the equal inclusion/exclusion criteria for ETV single treatment were screened, we randomly selected 87 patients with the ETV treatment duration matched with combined ADV and TDF treatment duration, and we designated this group as the ETV group (Fig. 1). The study protocol was approved by the Institutional Review Board of Seoul National University Hospital (IRB number: H-1603-065-748), which follows the ethical guidelines of the World Medical Association Declaration of Helsinki. Informed consent was waived because of the retrospective nature of the study.

\section{Selection of antiviral therapy}

The indication for antiviral therapy followed Korean reimbursement system, identical with the Korean Association for the Study of the Liver guideline, ${ }^{12}$ which also agrees with the recent guidelines of the American Association for the Study of Liver Diseases. ${ }^{13}$ Hepatitis B e antigen (HBeAg) positive CHB patients with inflammation and hepatitis $B$ DNA $\geq 20,000$, and $\mathrm{HBeAg}$ negative $\mathrm{CHB}$ patients with inflammation and hepatitis B DNA $\geq 2,000$ received antiviral treatment, and patients with cirrhosis had less strict standards. Patients treated with ADV based regimens were switched to TDF based regimens when viral suppression was insufficient or switched to TDF monotherapy due to compliance.

\section{Assessment of renal function}

GFR was calculated using the Modification of Diet in Renal Disease Study equation as the original study. ${ }^{14}$ Serial measurements of GFR, creatinine, and phosphorus were performed as each clinician's decision between 3 to 6 months. Changes of each parameter were measured retrospectively.

Definition of clinical manifestations of nephrotoxicity was defined as: (1) creatinine increase by at least $0.5 \mathrm{mg} / \mathrm{dL}$ over from baseline; (2) GFR decrease by at least 25\% over from baseline; (3) phosphorus decrease below $2.0 \mathrm{mg} / \mathrm{dL}$ with at least 0.5 $\mathrm{mg} / \mathrm{dL}$ decrease from baseline; (4) chronic kidney disease (CKD) ${ }^{15}$ stage 4 development with normal GFR from baseline; and (5) dose reduction of antiviral regimens from baseline.

\section{Statistical analysis}

To compare baseline characteristics, we used the t-test for continuous variables and the chi-square test for noncontinuous variables. The renal events between the ADV-TDF group and the ETV group were compared using the chi-square test, and for estimation of time factors, the Kaplan-Meier curve with

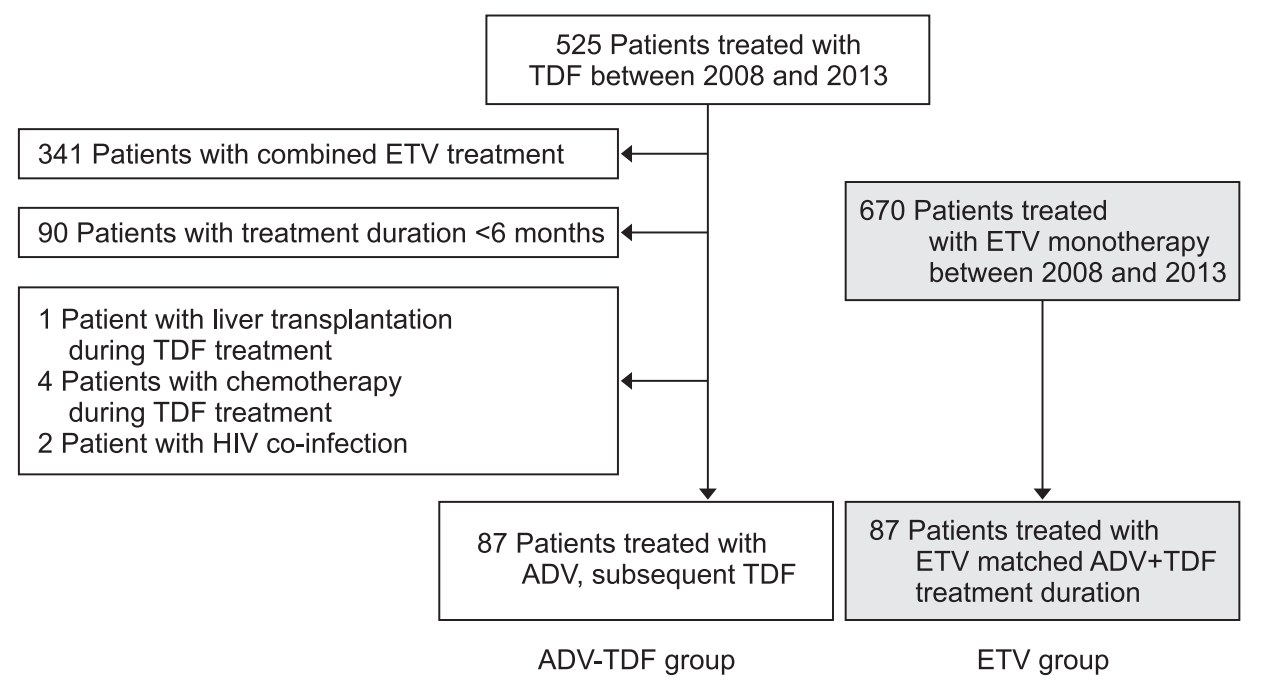

Fig. 1. Patient selection.

TDF, tenofovir; ETV, entecavir; HIV, human immunodeficiency virus; $\mathrm{ADV}$, adefovir. 
log-rank test for comparison was used. Prognostic factors were evaluated by univariate and multivariate analysis performed by the Cox proportional hazards model. To minimize selection bias, we used inverse probability weighting (IPW). Propensity scores were calculated using generalized boosted regression to predict the probability of each patient receiving ADF-TDF or ETV, using age, sex, diabetes, hypertension, liver cirrhosis, HBeAg seropositivity, and presence of HCC as pretreatment variables. After propensity scores were calculated, the two groups were balanced by means of IPW.

All statistical analyses were performed using SPSS Statistics, version 22.0 (IBM Corp., Armonk, NY, USA) and R Statistical Software 3.1 (R Foundation for Statistical Computing, Vienna, Austria), and a two-sided p-value $<0.05$ was considered statistically significant.

Table 1. Baseline Characteristics

\begin{tabular}{|c|c|c|c|}
\hline Characteristic & $\begin{array}{l}\text { ADV-TDF group } \\
\quad(\mathrm{n}=87)\end{array}$ & $\begin{array}{l}\text { ETV group } \\
(\mathrm{n}=87)\end{array}$ & $\mathrm{p}$-value \\
\hline Age, yr & $55.2 \pm 11.9$ & $57.8 \pm 9.45$ & 0.11 \\
\hline Male sex & $57(65.5)$ & $52(59.8)$ & 0.43 \\
\hline Diabetes & $9(10.3)$ & $6(6.9)$ & 0.41 \\
\hline Hypertension & $8(9.2)$ & $5(5.7)$ & 0.38 \\
\hline Liver cirrhosis & $28(32.2)$ & 65 (74.7) & $<0.01^{*}$ \\
\hline Log HBV DNA & $5.7 \pm 1.8$ & $5.6 \pm 1.3$ & 0.74 \\
\hline HBeAg negative & $22(25.3)$ & $61(70.1)$ & $<0.01^{*}$ \\
\hline Mutation & 85 (98.8) & 0 & NA \\
\hline Hepatocellular carcinoma & $13(14.9)$ & $16(18.4)$ & 0.54 \\
\hline ALT, IU/L & $82.1 \pm 132.1$ & $114.3 \pm 146.1$ & 0.12 \\
\hline Creatinine, $\mathrm{mg} / \mathrm{dL}$ & $0.90 \pm 0.25$ & $0.87 \pm 0.17$ & 0.33 \\
\hline GFR, $\mathrm{mL} / \mathrm{min} / 1.73 \mathrm{~m}^{2}$ & $87.9 \pm 21.2$ & $87.3 \pm 15.7$ & 0.82 \\
\hline Albumin, g/dL & $4.30 \pm 0.38$ & $4.06 \pm 0.49$ & $<0.01^{*}$ \\
\hline Bilirubin, mg/dL & $0.98 \pm 0.59$ & $1.38 \pm 2.73$ & 0.18 \\
\hline Albuminuria & & & 0.34 \\
\hline None & $18(20.7)$ & $21(24.1)$ & \\
\hline$+1-$ & $6(6.9)$ & $4(4.6)$ & \\
\hline $1+$ & $5(5.7)$ & $1(1.1)$ & \\
\hline Hematuria & & & 0.56 \\
\hline None & 26 (29.9) & $20(23.0)$ & \\
\hline$+1-$ & $2(2.3)$ & $3(3.4)$ & \\
\hline $1+$ & $1(1.1)$ & $3(3.4)$ & \\
\hline Missing & $58(66.7)$ & $61(70.1)$ & \\
\hline
\end{tabular}

Data are presented as mean \pm SD or number (\%).

$\mathrm{ADV}$, adefovir; TDF, tenofovir; ETV, entecavir; HBV, hepatitis B virus; HBeAg, hepatitis B e antigen; ALT, alanine aminotransferase; GFR, glomerular filtration rate; NA, not available. ${ }^{*} \mathrm{p}<0.01$.

\section{RESULTS}

\section{Baseline characteristics}

We selected 87 patients in the ADV-TDF group from a single center using the inclusion/exclusion criteria. Baseline characteristics were collected at the time of starting ADV treatment, patients were male predominant (65.5\%), and most patients had HBV mutation at baseline (98.8\%) (Table 1). The mutations were mostly lamivudine-associated mutations ( $n=84,96.6 \%$ ), and only one patient (1.1\%) had ADV-associated mutations. At baseline, 83 patients (95.4\%) were treated in combination with lamivudine, telbivudine, or clevudine. After changing to TDF based regimens 68 patients (78.2\%) maintained combination treatments and 19 patients (21.8\%) switched to TDF monotherapy. There were 670 patients treated with ETV monotherapy matching the inclusion/exclusion criteria, and we randomly matched these patients with the treatment duration. At baseline, both groups were well balanced in the renal function; creatinine, GFR, albuminuria, and hematuria (Table 1). Baseline demographics show that ADV treated patients have more radiological liver cirrhosis and HBeAg positivity compared to ETV treated patients (Table 1).

\section{Renal events of the ADV-TDF group compared to the ETV group}

The median duration of follow-up was 58.0 months (interquartile range, 41.0 to 69.0 months). During follow up, significantly more patients developed GFR decrease over 25\% in the ADV-TDF group compared to the ETV group (31.0\% vs 14.9\%, $\mathrm{p}=0.01$, respectively) (Table 2). Creatinine increase over 0.5 $\mathrm{mg} / \mathrm{dL}$ and phosphorus decrease below $2.0 \mathrm{mg} / \mathrm{dL}$ was more frequent in the ADV-TDF group compared to the ETV group, although not significant. Dose reduction was also more frequent in the ADV-TDF group compared to the ETV group (6.9\% vs 2.3\%, $\mathrm{p}=0.27$, respectively) (Table 2).

After analyzing time effects, the ADV-TDF group still showed significantly higher GFR decrease over 25\% compared to ETV

Table 2. Renal Events

\begin{tabular}{lccc}
\hline & $\begin{array}{c}\text { ADV-TDF } \\
\text { group } \\
(\mathrm{n}=87)\end{array}$ & $\begin{array}{c}\text { ETV } \\
\text { group } \\
(\mathrm{n}=87)\end{array}$ & p-value \\
\hline Cr increase by over 0.5 mg/dL & $5(5.7)$ & $2(2.3)$ & 0.44 \\
GFR decrease by over 25\% & $27(31.0)$ & $13(14.9)$ & $0.01^{*}$ \\
P decrease below 2.0 mg/dL & $13(14.9)$ & $5(5.9)$ & 0.05 \\
Developed CKD stage 4 & $2(2.3)$ & 0 & 0.49 \\
Dose reduction & $6(6.9)$ & $2(2.3)$ & 0.27 \\
\hline
\end{tabular}

Data are presented as number (\%).

$\mathrm{ADV}$, adefovir; TDF, tenofovir; ETV, entecavir; Cr, creatinine; GFR, glomerular filtration rate; $\mathrm{P}$, phosphorus; CKD, chronic kidney disease.

${ }^{*} \mathrm{p}<0.05$. 
group ( $\mathrm{p}=0.02$ by log-rank test) (Fig. $2 \mathrm{~B}$ ). Creatinine increase over $0.5 \mathrm{mg} / \mathrm{dL}$ ( $\mathrm{p}=0.25$ by log-rank test) (Fig. $2 \mathrm{~A}$ ) and phosphorus decrease below $2.0 \mathrm{mg} / \mathrm{dL}$ ( $\mathrm{p}=0.06$ by log-rank test) (Fig. $2 \mathrm{C}$ ) were not different between the ADV-TDF group and the ETV group. Cox regression analysis revealed no factors associated with creatinine increase over $0.5 \mathrm{mg} / \mathrm{dL}$, the ADV-TDF group versus the ETV group (hazard ratio [HR], 2.15; 95\% confidential interval [CI], 1.10 to 4.18 ; $p=0.02$ ) (Table 3) was associated with GFR decrease over 25\% after adjusting for decrease of baseline GFR under $90 \mathrm{~mL} / \mathrm{min} / 1.73 \mathrm{~m}^{2}$. The presence of HCC was the only factor associated with phosphorus decrease below $2.0 \mathrm{mg} /$ dL (HR, 5.65; 95\% CI, 2.22 to 14.36; p<0.01) (Table 3).

Liver cirrhosis and $\mathrm{HBeAg}$ seropositivity may act as a confounder of renal events. Therefore, we performed univariate cox regression analysis stratified by liver cirrhosis, and $\mathrm{HBeAg}$ seropositivity. The Forest plot shows tendency to favor GFR decrease in the ADV-TDF group compared to the ETV group in all subgroups, although it was only statistically significant in the HBeAg negative subgroup (Fig. 3). The lack of statistical significance may be due to the small sample size in the subgroups We also performed IPW analysis to overcome the difference of baseline characteristics. After balancing the two groups using propensity score, the ADV-TDF group was well balanced to the ETV group (Supplementary Table 1). Log-rank analysis of the weighted group showed similar results compared to the unweighted group. The ADV-TDF group showed higher risk of renal events compared to the ETV group only in the analysis of GFR decrease over 25\% ( $\mathrm{p}=0.047$ by log-rank test) (Supplementary Fig. 1).
A

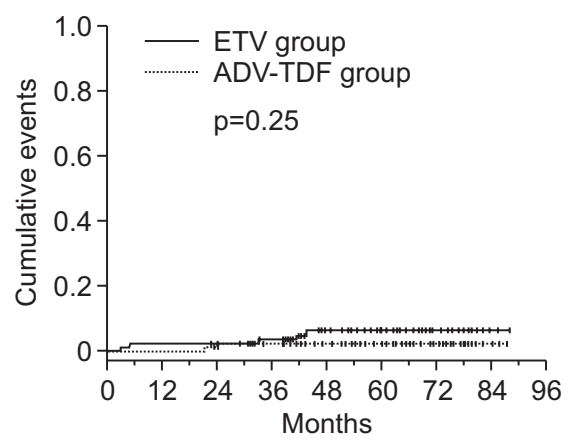

B

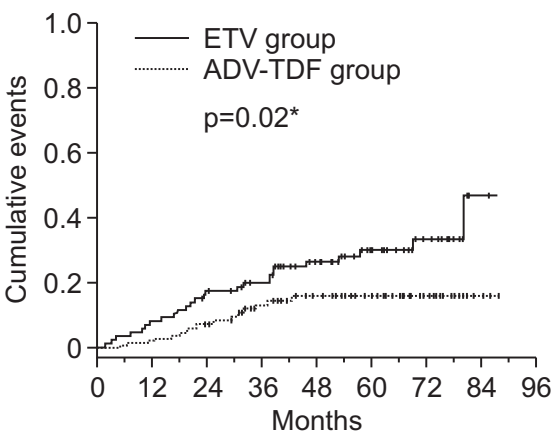

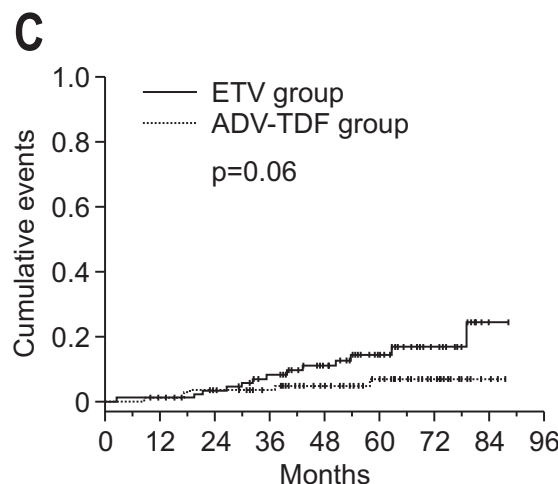

Fig. 2. Renal events in the ADV-TDF group compared to the ETV group. (A) Kaplan-Meier curve of the creatinine increase over $0.5 \mathrm{mg} / \mathrm{dL}$. (B) Kaplan-Meier curve of the GFR decrease greater than 25\%. (C) Kaplan-Meier curve of the phosphorus decrease below $2.0 \mathrm{mg} / \mathrm{dL}$. $\mathrm{ADV}$, adefovir; TDF, tenofovir; ETV, entecavir; GFR, glomerular filtration rate. ${ }^{*} \mathrm{p}<0.05$.

Table 3. Risk Factors for Renal Impairment in the ADV-TDF Group Compared to Those in the ETV Group

\begin{tabular}{|c|c|c|c|c|c|c|}
\hline \multirow{2}{*}{ Variable } & \multicolumn{2}{|c|}{ Cr increase over $0.5 \mathrm{mg} / \mathrm{dL}$} & \multicolumn{2}{|c|}{ GFR decrease over 25\% } & \multicolumn{2}{|c|}{$\mathrm{P}$ decrease below $2.0 \mathrm{mg} / \mathrm{dL}$} \\
\hline & HR $(95 \%$ CI) & p-value & HR $(95 \%$ CI) & p-value & HR $(95 \%$ CI) & $\mathrm{p}$-value \\
\hline Male sex & $0.26(0.03-2.14)$ & 0.21 & $0.59(3.14-1.10)$ & 0.10 & $0.19(0.04-0.84)$ & 0.05 \\
\hline Age & $1.07(0.98-1.16)$ & 0.10 & $1.02(0.99-1.05)$ & 0.13 & $1.04(0.99-1.09)$ & 0.08 \\
\hline Diabetes & Failure to predict model & & $1.61(0.63-4.14)$ & 0.31 & $2.38(0.69-8.25)$ & 0.17 \\
\hline Liver cirrhosis & $2.21(0.43-11.4)$ & 0.34 & $0.90(0.48-1.69)$ & 0.74 & $1.10(0.43-2.78)$ & 0.85 \\
\hline Log HBV DNA & $0.86(0.55-1.32)$ & 0.48 & $1.08(0.88-1.33)$ & 0.44 & $0.84(0.65-1.11)$ & 0.22 \\
\hline Hepatocellular carcinoma & $2.08(0.40-10.8)$ & 0.38 & $1.83(0.89-3.77)$ & 0.09 & $5.65(2.22-14.36)$ & $<0.01^{\dagger}$ \\
\hline $\begin{array}{l}\text { Baseline GFR } \\
\qquad>90 \mathrm{~mL} / \mathrm{min} / 1.73 \mathrm{~m}^{2}\end{array}$ & Reference & & Reference & & Reference & \\
\hline $\begin{array}{l}\text { Baseline GFR } \\
\leq \leq 90 \mathrm{~mL} / \mathrm{min} / 1.73 \mathrm{~m}^{2}\end{array}$ & $0.79(0.15-4.06)$ & 0.77 & $3.67(1.92-7.00)$ & $<0.01^{\dagger}$ & $1.93(0.63-5.87)$ & 0.24 \\
\hline ETV group & Reference & & Reference & & Reference & \\
\hline ADV-TDF group & $2.52(0.49-13.0)$ & 0.26 & $2.10(1.08-4.10)$ & $0.03^{*}$ & $2.53(0.90-7.12)$ & 0.08 \\
\hline
\end{tabular}

$\mathrm{ADV}$, adefovir; TDF, tenofovir; ETV, entecavir; Cr, creatinine; GFR, glomerular filtration rate; P, phosphorus; HR, hazard ratio; CI, confidence interval; $\mathrm{HBV}$, hepatitis B virus.

${ }^{*} \mathrm{p}<0.05 ;{ }^{\dagger} \mathrm{p}<0.01$. 


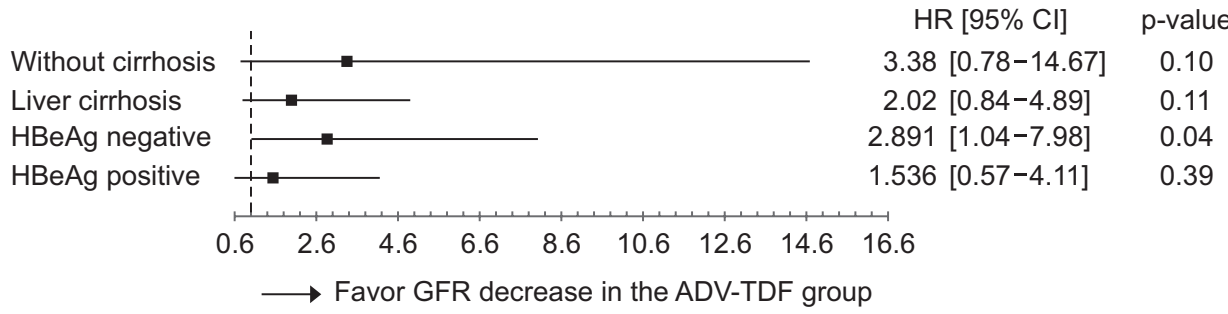

Fig. 3. Univariate analysis of GFR decrease greater than 25\% stratified by liver cirrhosis and HBeAg seropositivity.

GFR, glomerular filtration rate; $\mathrm{HBeAg}$, hepatitis B e antigen; ADV, adefovir; TDF, tenofovir; HR, hazard ratio; 95\% CI, confidence interval.

\section{DISCUSSION}

This study aimed to compare the long-term renal adverse event between the ADV-TDF group and the ETV group. We have shown that long-term sequential nucleotide analogue treatment is associated with significant GFR decrease. Although not statistically significant, the ADV-TDF group patients had decreased phosphorus levels compared to the ETV group patients, suggesting that tubular injury might be a possible cause of renal adverse events.

There are limited data regarding the occurrence of renal adverse events of nucleotide analogues in CHB patients. The results of our study suggest there are significant renal adverse events in the long-term nucleotide analogue treated groups, and we need to consider an early switch to other regimens such as nucleoside analogue or the novel nucleotide analogue with less renal events, tenofovir alafenamide. ${ }^{16}$

Complete cure of CHB is thought to be as eliminating closely covalent circular DNA from the liver. There are novel therapies in pursuit for a complete cure, but it is not possible with current treatment regimens. The practical cure of CHB is HBsAg seroclearance, a goal which is possible, but a very little proportion of patients reach. ${ }^{17}$ Therefore, most guidelines recommend longterm use of NAs and indefinite treatment in some indications. ${ }^{13}$ Previously, in the era of low potency antivirals, the most important problem of NA treatment was the evolution of drug mutations after long-term use of NAs. However, this problem is not an important issue in current high genetic barrier NA treatments such as ETV and TDF. The most important concern of NA treatment is currently long-term complications such as renal adverse events and bone mineral density loss. These complications are well known and studied in HIV infected patients, but there were controversies in CHB patients.

A previous American study compared renal adverse events using serum creatinine increase and GFR decrease between TDF treated patients and ETV treated CHB patients, concluding that there was a comparable risk between the groups. ${ }^{5}$ However, the median follow-up of TDF treatment period was only 80 weeks. A recent Turkish multicenter study analyzed a large cohort of antiviral treated CHB patients from 22 centers. ${ }^{18}$ This study evaluated renal adverse events till 24 months of follow-up, TDF caused decreased GFR, but the difference was not clinically significant. Another recent Spanish multicenter study followed-up for 49 months evaluating renal functions were stable with TDF and ETV treatment. However, renal adverse events were not the primary outcome of the study. ${ }^{19}$

The strength of this study is the long-term follow-up period of nucleotide analogue treatment of median 60 months. There are very few studies evaluating the long-term toxicities of nucleotide analogues. Studies in HIV positive patients undergoing TDF treatment have shown there is a significant time for the occurrence of renal adverse events. A previous American study has shown that a significant progression to CKD stage 3 occurs between year 4 and year 5 after TDF treatment. ${ }^{11}$ The finding of this previous study implies long-term follow-up is mandatory to evaluate renal adverse events.

We merged the treatment periods of ADV and TDF, as the ADV-TDF groups. Renal adverse events of ADV have been studied more than those of TDF. However, as the renal toxicity of high dose ADV treatment is well known, clinicians make timely dose adjustments when treating $\mathrm{ADV}$ for $\mathrm{CHB}$ patients. ${ }^{20} \mathrm{~A}$ previous long-term study on $\mathrm{CHB}$ patients treated by ADV or TDF followed-up for mean 7.4 years. ${ }^{10}$ The mean time to onset of renal tubular dysfunction was 49 months with a 10-year cumulative rate of $15 \%$. One study also concluded that TDF has similar renal adverse events regardless of previous ADV treatment. ${ }^{21}$ TDF and ADV also share the phenotypes of renal adverse events such as hypophosphatemia and osteomalacia. ${ }^{10}$ The two drugs share a similar structure, and the mechanisms of renal toxicities are similar, thereby it is theoretically sound to combine the two different treatments.

The risk factors of renal tubular dysfunction in the treatment of ADV or TDF in CHB patients were old age and low baseline GFR. ${ }^{10}$ Other previous studies have found old age, diuretics treatment as risk factors for renal adverse events of antiviral treatment on CHB patients. ${ }^{22,23}$ The data of this study show that only the ADV-TDF group was at a risk of GFR decrease, as more number of patients in this group showed a decrease in phosphorus; tubular dysfunction could be a possible mechanism of the GFR decrease. Presence of HCC was the only risk factor for phosphorus decrease. Cancer itself could cause hypophosphatemia, ${ }^{24}$ and chemotherapeutic agents such as sorafenib might also cause hypophosphatemia in HCC patients. ${ }^{25}$

The limitations of this study are the retrospective nature of the design. There were significant differences in baseline characteristics, such as liver cirrhosis, HBeAg positivity, and baseline 
HBV virus mutations between the two groups. This baseline difference was inevitable from the study design. However, these factors were not associated with renal adverse events in CHB patients in previous studies. We tried to overcome the difference of baseline characteristics by subgroup analysis and IPW analysis.

In conclusion, the ADV-TDF group showed significantly higher renal events compared to the ETV group. Clinicians should be aware of renal toxicity development, and further long-term study needs to be performed.

\section{CONFLICTS OF INTEREST}

J.H.Y. has received a research grant from Bayer HealthCare Pharmaceuticals; Y.J.K. has received research grants from Bristol-Myers Squibb, Roche, JW CreaGene, Bukwang Pharmaceuticals, Handok Pharmaceuticals, Hanmi Pharmaceuticals, Yuhan Pharmaceuticals, and Pharmaking, and has received lecture fees from Bayer HealthCare Pharmaceuticals, Gilead Science, MSD Korea, Yuhan Pharmaceuticals, Samil Pharmaceuticals, CJ Pharmaceuticals, Bukwang Pharmaceuticals, and Handok Pharmaceuticals; and S.J.Y. has received a lecture fee from Bayer HealthCare Pharmaceuticals. The other authors declare that they have no conflicting interests.

\section{ACKNOWLEDGEMENTS}

This research was supported by a grant of the Korea Health Technology R\&D Project through the Korea Health Industry Development Institute (KHIDI), funded by the Ministry of Health \& Welfare, Republic of Korea (grant number: HI16C1074) and the Daewoong Corporation (grant number: 0620161740 [20161766]).

\section{AUTHOR CONTRIBUTIONS}

Study concept and design: Y.J.K. Data analysis: Y.Y.C. Data acquisition: Y.C., J.Y.N., H.C. Statistical analysis: Y.C., J.Y.N., H.C. Drafting the manuscript: Y.Y.C. Critical revision: E.J.C., J.H.L., S.J.Y., J.H.Y. Approval of final manuscript: all authors.

\section{ORCID}

Young Youn Cho Young Chang Joon Yeul Nam Hyeki Cho Eun Ju Cho Jeong-Hoon Lee Su Jong Yu Jung-Hwan Yoon Yoon Jun Kim https://orcid.org/0000-0002-9384-5357 https://orcid.org/0000-0001-7752-7618 https://orcid.org/0000-0003-4471-5529 https://orcid.org/0000-0003-3694-8111 https://orcid.org/0000-0002-2677-3189 https://orcid.org/0000-0002-0315-2080 https://orcid.org/0000-0001-8888-7977 https://orcid.org/0000-0002-9128-3610 https://orcid.org/0000-0001-9141-7773

\section{REFERENCES}

1. Lee WM. Hepatitis B virus infection. N Engl J Med 1997;337:17331745.

2. Cho EJ, Kim SE, Suk KT, et al. Current status and strategies for hepatitis B control in Korea. Clin Mol Hepatol 2017;23:205-211.

3. Lok AS, McMahon BJ, Brown RS Jr, et al. Antiviral therapy for chronic hepatitis B viral infection in adults: a systematic review and meta-analysis. Hepatology 2016;63:284-306.

4. U.S. National Library of Medicine. Dailymed, archived drug label [Internet]. Bethesda, NLM; c2018 [cited 2019 Jul 7]. Available from: https://dailymed.nlm.nih.gov/dailymed/archives/fdaDrugInfo.cfm?archiveid=10048.

5. Gish RG, Clark MD, Kane SD, Shaw RE, Mangahas MF, Baqai S. Similar risk of renal events among patients treated with tenofovir or entecavir for chronic hepatitis B. Clin Gastroenterol Hepatol 2012;10:941-946.

6. Hall AM, Hendry BM, Nitsch D, Connolly JO. Tenofovir-associated kidney toxicity in HIV-infected patients: a review of the evidence. Am J Kidney Dis 2011;57:773-780.

7. Hwang HS, Park CW, Song MJ. Tenofovir-associated Fanconi syndrome and nephrotic syndrome in a patient with chronic hepatitis B monoinfection. Hepatology 2015;62:1318-1320.

8. Cho H, Cho Y, Cho EJ, et al. Tenofovir-associated nephrotoxicity in patients with chronic hepatitis B: two cases. Clin Mol Hepatol 2016;22:286-291.

9. Tanji N, Tanji K, Kambham N, Markowitz GS, Bell A, D’agati VD. Adefovir nephrotoxicity: possible role of mitochondrial DNA depletion. Hum Pathol 2001;32:734-740.

10. Gara N, Zhao X, Collins MT, et al. Renal tubular dysfunction during long-term adefovir or tenofovir therapy in chronic hepatitis $\mathrm{B}$. Aliment Pharmacol Ther 2012;35:1317-1325.

11. Monteagudo-Chu MO, Chang MH, Fung HB, Bräu N. Renal toxicity of long-term therapy with tenofovir in HIV-infected patients. J Pharm Pract 2012;25:552-559.

12. Korean Association for the Study of the Liver. KASL clinical practice guidelines: management of chronic hepatitis B. Clin Mol Hepatol 2016;22:18-75.

13. Terrault NA, Bzowej NH, Chang KM, et al. AASLD guidelines for treatment of chronic hepatitis B. Hepatology 2016;63:261-283.

14. Levey AS, Stevens LA, Schmid CH, et al. A new equation to estimate glomerular filtration rate. Ann Intern Med 2009;150:604612.

15. Inker LA, Astor BC, Fox CH, et al. KDOQI US commentary on the 2012 KDIGO clinical practice guideline for the evaluation and management of CKD. Am J Kidney Dis 2014;63:713-735.

16. Abdul Basit S, Dawood A, Ryan J, Gish R. Tenofovir alafenamide for the treatment of chronic hepatitis B virus infection. Expert Rev Clin Pharmacol 2017;10:707-716.

17. Wu D, Ning Q. Toward a cure for hepatitis B virus infection: combination therapy involving viral suppression and immune modulation and long-term outcome. J Infect Dis 2017;216:S771-S777. 
18. Koklu S, Gulsen MT, Tuna Y, et al. Differences in nephrotoxicity risk and renal effects among anti-viral therapies against hepatitis B. Aliment Pharmacol Ther 2015;41:310-319.

19. Riveiro-Barciela M, Tabernero D, Calleja JL, et al. Effectiveness and safety of entecavir or tenofovir in a Spanish cohort of chronic hepatitis b patients: validation of the page-B score to predict hepatocellular carcinoma. Dig Dis Sci 2017;62:784-793.

20. Hartono JL, Aung MO, Dan YY, et al. Resolution of adefovirrelated nephrotoxicity by adefovir dose-reduction in patients with chronic hepatitis B. Aliment Pharmacol Ther 2013;37:710-719.

21. Marengo A, Marietti M, Rizzetto M, Marzano A. Letter: renal effects of tenofovir in adefovir dipivoxil-experienced HBV patients. Aliment Pharmacol Ther 2014;40:324.
22. Tsai MC, Chen CH, Tseng PL, et al. Comparison of renal safety and efficacy of telbivudine, entecavir and tenofovir treatment in chronic hepatitis B patients: real world experience. Clin Microbiol Infect 2016;22:95.

23. Kim YJ, Cho HC, Sinn DH, et al. Frequency and risk factors of renal impairment during long-term adefovir dipivoxil treatment in chronic hepatitis B patients. J Gastroenterol Hepatol 2012;27:306312.

24. Stewart AF. Clinical practice. Hypercalcemia associated with cancer. N Engl J Med 2005;352:373-379.

25. Llovet JM, Ricci S, Mazzaferro V, et al. Sorafenib in advanced hepatocellular carcinoma. N Engl J Med 2008;359:378-390. 